\title{
Novel Behaviors to the Nonlinear Evolution Equation Describing the Dynamics of Ionic Currents along Microtubules
}

\author{
Haci Mehmet Baskonus ${ }^{1, \star}$, Fevzi Erdogan ${ }^{2, \star \star}$, Arif Ozkul ${ }^{3, \star \star \star}$, and Ilham Asmouh ${ }^{4, \star \star \star \star}$ \\ ${ }^{1}$ Department of Computer Engineering, Munzur University, Tunceli, Turkey \\ ${ }^{2}$ Department of Mathematics, Yuzuncu Yil University, Van, Turkey \\ ${ }^{3}$ Department of Mathematics, Firat University, Elazig, Turkey \\ ${ }^{4}$ Department of Mathematics and Applications, Abdelmalek Essaadi University, Fas
}

\begin{abstract}
In this work, we consider the Bernoulli sub-equation function method for obtaining novel behaviors to the nonlinear evolution equation describing the dynamics of ionic currents along Microtubules. We obtain new results by using this technique. We plot two- and three-dimensional surfaces of the results by using Wolfram Mathematica 9 . At the end of this manuscript, we submit a conclusion in the comprehensive manner.
\end{abstract}

\section{Introduction}

For the past two decades, searching for the solutions to the various nonlinear evolution equations have became a great activities between scientists in the world. Nonlinear evolution equations arise in the various fields of nonlinear sciences such as optical fibers, chemistry, biology, fluid mechanics etc, and their solutions play a vital roles in our real life situations. Various powerful techniques have been developed for the study of the behavior to the different nonlinear evolution equations such as the $\left(G^{\prime} / G\right)$-expansion method [1], the new extended $\left(G^{\prime} / G\right)$-expansion method [2], the sine-cosine method [3], the sine-Gordon expansion method [4, 5], the tanh method [6, 7], the extended tanh method [8], the Hirota bilinear method [9], the modified simple equation method [10], the modified tanh-coth method [11], the Riccati-Bernoulli sub-ODE method [12] and so on. In general, various powerful techniques have been developed by various researchers to tackle different class of nonlinear evolution equations [13-17].

However, in this article, we explore the search for the new solutions to the transmission line models of nano-ionic currents along microtubules $[18,19]$ by using the Bernoulli sub-equation function method (BSEFM) [20].

The transmission line models of nano-ionic currents along microtubules is given by $[18,19]$ :

$$
w L^{2} u_{x x t}+L^{2} u_{x x}+2 \kappa \delta u u_{t}-\kappa u_{t}=0
$$

\footnotetext{
^e-mail: hmbaskonus@gmail.com

$\star \star$ e-mail: fevzier@gmail.com

$\star \star \star$ e-mail: arifozkul@outlook.com

$\star \star \star \star$ e-mail: ilham.fst@gmail.com
} 


\section{Analysis of the Method}

In this section, we present the steps to be followed for the Bernoulli sub-equation function method in searching new solutions to various nonlinear evolution equations.

Step-1. We consider the partial differential equation in two variables such as $x, t$ and a dependent variable $u$;

$$
P\left(u_{x}, u_{t}, u_{x x}, u_{x t}, \ldots\right),
$$

and take the wave transformation

$$
u(x, t)=U(\zeta), \zeta=x-k t,
$$

where $k \neq 0$. Substituting Eq. (3) into Eq. (2), it gives us the following nonlinear ordinary differential equation;

$$
N\left(U, U^{\prime}, U^{\prime \prime}, U^{\prime \prime \prime}, \ldots\right)
$$

textbfStep-2. Take trial equation as follows

$$
U(\zeta)=\sum_{i=0}^{n} a_{i} F^{i}(\zeta)=a_{0}+a_{1} F(\zeta)+a_{2} F^{2}(\zeta)+\ldots+a_{n} F^{n}(\zeta)
$$

in which

$$
F^{\prime}=b F(\zeta)+d F^{M}(\zeta)
$$

where $b \neq 0, d \neq 0, M \in \mathbb{R} \backslash\{0,1,2\}$, and $F(\zeta)$ is Bernoulli differential polynomial. Substituting above relations into Eq. (4), we obtain an equation of polynomial $\Omega(F(\zeta))$ of $F(\zeta)$;

$$
\Omega(F(\zeta))=\rho_{s} F^{s}(\zeta)+\ldots+\rho_{1} F(\zeta)+\rho_{0}=0 .
$$

According to the balance principle, we can get values of and $M$ and $n$.

Step-3. Let us consider the coefficients of $\Omega(F(\zeta))$ all be zero, we will obtain an algebraic equations system:

$$
\rho_{i}=0, \quad i=0, \ldots, s .
$$

Solving this system, we will determine the values of $a_{0}, \ldots a_{n}$.

Step-4. When we solve nonlinear Bernoulli differential equation that is Eq. (6), we obtain the following two situations according to $b$ and $d$;

$$
\begin{gathered}
F(\zeta)=\left[\frac{-d}{b}+\frac{c}{e^{b(M-1) \zeta}}\right]^{\frac{1}{1-M}}, \quad b \neq d \\
F(\zeta)=\left[\frac{(c-1)+(c+1) \tanh \left(\frac{b(1-M) \zeta}{2}\right)}{1-\tanh \left(\frac{b(1-M) \zeta}{2}\right)}\right]^{\frac{1}{1-M}}, \quad b=d, c \in \mathbb{R} .
\end{gathered}
$$


Using the complete discrimination of the polynomial of $F$, we solve the system of equations by using the Wolfram Mathematica 9 and obtain the various cases for the results of the coefficients. We obtain the exact solutions to Eq. (2) by putting the obtained values of the coefficients into the trial solution that is Eq. (5).

\section{Application}

In this section, we apply the BSEFM to the transmission line models of nano-ionic currents along microtubules [18, 19] given in Eq. (1).

Utilizing the wave transformation $u=U(\zeta) \zeta=k x-c t$ on Eq. (1), yields;

$$
-c k^{2} w L^{2} U^{\prime \prime}+L^{2} k^{2} U^{\prime}-c \kappa \delta U^{2}+c \kappa U=0,
$$

applying the homogeneous balancing technique on Eq. (11) by using the highest derivative $U^{\prime \prime}$ and the highest power nonlinear term $U^{2}$, yields the following relation between $n$ and $M$;

$$
n=M+1 \quad n, M \in \mathbb{Z}^{+} .
$$

Choosing $M=3$, gives $n=4$. Using $n=4$ along with Eq. (5), yields the following;

$$
U(\zeta)=a_{0}+a_{1} F+a_{2} F^{2}+a_{3} F^{3}+a_{4} F^{4},
$$

differentiating Eq. (13) twice, produces the following equations;

$$
\begin{gathered}
U^{\prime}=a_{1} b F+a_{1} d F^{M}+2 a_{2} b F^{2}+2 a_{2} d F^{M+1}+3 a_{3} F^{3} \\
+3 a_{3} d F^{M+2}+4 a_{4} b F^{4}+4 a_{4} d F^{M+3}, \\
U^{\prime \prime}=a_{1} b F^{\prime}+a_{1} d F^{M-1} F^{\prime}+4 a_{2} b F F^{\prime}+16 a_{4} d F^{3} F^{\prime}+2 a_{2} d(M+1) F^{M} F^{\prime} \\
+9 a_{3} b F^{2} F^{\prime}+3(M+2) a_{3} F^{M+1} F^{\prime}+4 a_{4} d(M+3) F^{M+2} F^{\prime},
\end{gathered}
$$

where $F^{\prime}=b F+d F^{3}, b \neq 0, d \neq 0$. Substituting Eq. (14) and (15) into Eq. (11) and simplifying, we get an equation that involves the polynomials of $F$. We therefore collect the system of equations from that polynomial there by equating the summation of each coefficients of $F$ that are having the same power to zero. We simplify the system of equations with the help of Wolfram Mathematica 9 and get the following cases of solutions;

Case-1. when $b \neq d$

$a_{0}=\frac{a_{4} b^{2}}{d^{2}}, a_{1}=0, a_{2}=\frac{2 a_{4} b}{d}, a_{3}=0, w=-\frac{1}{10 b c}, \delta=\frac{d^{2}}{a_{4} b^{2}}, \kappa=\frac{12 b k^{2} L^{2}}{5 c}$,

substituting these values into Eq. (13), we get the following exponential function solution;

$$
u_{1}(x, t)=\frac{a_{4} b^{4} E^{2}}{d^{2}\left(d e^{-2 b c t+2 b k x-b E}\right)^{2}} .
$$



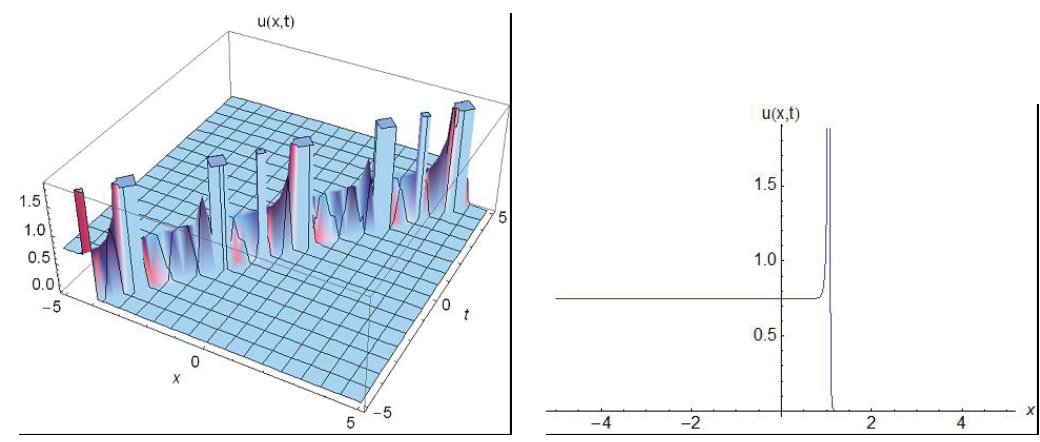

Figure 1. The 3D and 2D shapes of Eq. (16) with the values $k=6, w=2, b=2, E=8, c=5, d=4, a_{4}=3$, $-5<x<5,-5<t<5$ and $t=1.2$ for the $2 \mathrm{D}$ graphic.

Case-2. when $b \neq d$

$a_{0}=0, a_{1}=0, a_{2}=\frac{2 a_{4} b}{d}, a_{3}=0, w=-\frac{1}{10 b c}, \delta=-\frac{d^{2}}{a_{4} b^{2}}, \kappa=-\frac{12 b k^{2} L^{2}}{5 c}$,

substituting these values into Eq. (13), we get the following exponential function solution;

$$
u_{2}(x, t)=\frac{\left(-1+\frac{2 b E e^{2 b c t-b k x}}{d}\right) a_{4}}{\left(\frac{d}{b}-E e^{2 b c t-2 b k x}\right)^{2}} .
$$
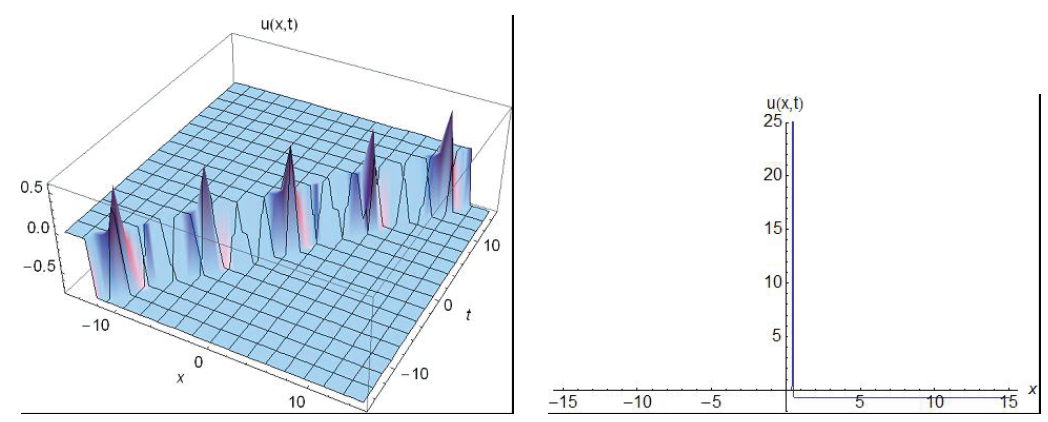

Figure 2. The 3D and 2D shapes of Eq. (17) with the values $k=6, w=2, b=2, E=8, c=5, d=4, a_{4}=3$, $-15<x<15,-15<t<15$ and $t=0.5$ for the $2 \mathrm{D}$ graphic.

\section{Conclusions}

In this study, with the help of Wolfram Mathematica 9, the Bernoulli sub-equation function method is employed in investigating some new behavior to a nonlinear evolution equation that describe the dynamics of ionic currents along Microtubules. We obtained some new exponential function structure to the model by utilizing the above mentioned method. All the obtained analytical solutions have 
satisfied the model. When we compare our obtained results with the results in the literature $[18,19]$ we observed that our result are newly constructed with the different solution structure. Therefore, with our available results, we can say that the Bernoulli sub-equation function method is simple and efficient mathematical tool that can be employed to various mathematical models.

\section{References}

[1] A Bekir and F Uygun, Arab Journal of Mathematical Sciences 18, 73-85 (2012)

[2] H Or-Roshid, MA Akbar, MdF Hoque and N Rahman, SpringerPlus 3(122), doi: 10.1186/21931801-3-122 (2014)

[3] H Zedan, SJ Monaquel, Applied Mathematics E-Notes 10, 103-111 (2010)

[4] H Bulut, TA Sulaiman and HM Baskonus, Opt. Quant. Electron 48(564), 1-14 (2016)

[5] H Bulut, TA Sulaiman, HM Baskonus and AA Sandulyak, Optik 135, 327-336 (2017)

[6] AH Khater, W Malfliet, DK Callebaut and ES Kamel, Chaos, Solitons and Fractals 14(3), 513-522 (2002)

[7] M Karimi, Mathematics Scientific Journal 9(1), 47-54 (2013)

[8] EME Zayed and HMA Rahman, Applied Mathematics E-Notes 10, 235-245 (2010)

[9] Z Jin-Ming and Z Yao-Ming, Chin. Phys. B 20(1), 010205 (2011)

[10] M Mirzazadeh, Information Sciences Letters 3(1), 1-9 (2014)

[11] L Wazzan, Communications in Nonlinear Science and Numerical Simulation 14(6), 2642-2652 (2009)

[12] X. Yang, Z Deng and Y Wei, Advances Differential Equations 117, DOI 10.1186/s13662-0150452-4 (2015)

[13] HM Baskonus and M Askin, $6^{\text {th }}$ International Youth Science Forum "LITTERIS ET ARTIBUS"' Computer Science and Engineering, Lviv, Ukraine, 24-26 November, (2016)

[14] MF El-Sabbagh, R Zait and RM Abdelazeem, Journal of Mathematics 10(4), 61-68 (2014)

[15] IE Inan and D Kaya, Physica A 381, 104-115 (2007)

[16] M Akbari, Computational Methods for Differential Equations 2(1), $50-55$ (2014)

[17] HM Baskonus, H Bulut, and M Kayhan, International Conference on Pure and Applied Mathematics (ICPAM-2015) Van/ Turkey,- 25-28 August (2015)

[18] MdN Alam and MdN Alam, Journal of Taibah University for Science (2017)

[19] E Tala-Tebue, ZI Djoufack, DC Tsobgni-Fozap, A Kenfack-Jiotsa, F Kapche-Tagne and TC Kofane, Chinese Journal of Physics doi: 10.1016/j.cjph.2017.03.004 (2017)

[20] B Zheng, WSEAS Transactions on Mathematics 7(11), 618-626 (2012) 